\title{
Estimation of Cold Flow Properties of Biodiesel from Fatty Acid Composition
}

\section{Supporting information (SI)}

Table S1. FAME composition (mass \%) and CFP (CFPP, CP and PP) of biodiesel feedstocks collected from the literature.

\begin{tabular}{|c|c|c|c|c|c|c|c|c|c|c|c|c|c|c|c|c|c|c|c|c|c|c|c|c|c|c|c|}
\hline$\#$ & Biodiesel & $\begin{array}{l}6: \\
0\end{array}$ & $8: 0$ & $10: 0$ & $12: 0$ & $14: 0$ & $14: 1$ & $15: 0$ & $16: 0$ & $16: 1$ & $17: 0$ & $17: 1$ & $18: 0$ & $18: 1$ & $18: 2$ & $18: 3$ & $20: 0$ & $20: 1$ & $20: 2$ & $20: 4$ & $22: 0$ & $22: 1$ & $24: 0$ & $24: 1$ & CFPP & $\mathrm{CP}$ & PP \\
\hline 1 & Almond ${ }^{1}$ & 0 & 0 & 0 & 0 & 0 & 0 & 0 & 10.4 & 0.5 & 0 & 0 & 2.9 & 77.1 & 7.6 & 0.8 & 0.3 & 0 & 0 & 0 & 0.1 & 0 & 0.2 & 0.4 & -6 & - & - \\
\hline 2 & $\begin{array}{l}\text { Aphanamixis } \\
\text { polystachya }\end{array}$ & 0 & 0 & 0 & 0 & 0 & 0 & 0 & 18.4 & 0.3 & 0 & 0 & 11.8 & 18.3 & 26.7 & 23.2 & 0.5 & 0.2 & 0 & 0 & 0 & 0.6 & 0 & 0 & - & 8 & 8 \\
\hline 3 & Argania spinosa 3 & 0 & 0 & 0 & 0 & 0.1 & 0 & 0 & 12.5 & 1.2 & 0 & 0 & 5.9 & 43.6 & 35.8 & 0.3 & 0.4 & 0 & 0 & 0 & 0.2 & 0 & 0 & 0 & 4.5 & 8 & 5 \\
\hline 4 & Bovine Tallow ${ }^{4}$ & 0 & 0 & 0 & 0 & 5.1 & 0 & 1.2 & 25 & 3.8 & 3.1 & 0 & 22.8 & 34.5 & 2.6 & 0 & 1.4 & 0 & 0 & 0 & 0 & 0 & 0 & 0 & 15 & - & - \\
\hline 5 & $\begin{array}{l}\text { Calophyllum } \\
\text { inophyllum5 }\end{array}$ & 0 & 0 & 0 & 0 & 0 & 0 & 0 & 15 & 0.4 & 0 & 0 & 16.7 & 39.1 & 26.9 & 0.6 & 0.9 & 0.3 & 0 & 0 & 0.4 & 0 & 0 & 0 & 12 & 14 & 13 \\
\hline 6 & $\begin{array}{l}\text { Calophyllum } \\
\text { inophyllum }\end{array}$ & 0 & 0 & 0 & 0 & 0 & 0 & 0 & 14.8 & 0.3 & 0 & 0 & 16 & 41.3 & 26.6 & 0.2 & 0.8 & 0 & 0 & 0 & 0 & 0 & 0 & 0 & - & 10 & 8 \\
\hline 7 & Camelina sativa ${ }^{7}$ & 0 & 0 & 0 & 0 & 0 & 0 & 0 & 5.4 & 0 & 0 & 0 & 2.6 & 14.3 & 14.3 & 38.4 & 0.3 & 16.8 & 0 & 0 & 1.4 & 2.9 & 0 & 0 & -4 & - & -8 \\
\hline 8 & Canola $^{8}$ & 0 & 0 & 0 & 0 & 0 & 0 & 0 & 4.5 & 0.3 & 0 & 0 & 2.2 & 62.7 & 20.6 & 9.7 & 0 & 0 & 0 & 0 & 0 & 0 & 0 & 0 & -12 & -1.2 & 10.3 \\
\hline 9 & Canola $^{9}$ & 0 & 0 & 0 & 0 & 0.1 & 0 & 0 & 4.1 & 0.2 & 0 & 0 & 1.9 & 58.9 & 20.6 & 9.3 & 0.6 & 1.4 & 0 & 0 & 0.4 & 0.1 & 0 & 0 & -8 & - & - \\
\hline 10 & Chicken Fat ${ }^{10}$ & 0 & 0 & 0 & 0 & 0.6 & 0.1 & 0 & 23.8 & 7.2 & 0.3 & 0.1 & 5.6 & 43.6 & 16.8 & 0.8 & 0.1 & 0.3 & 0 & 0 & 0 & 0 & 0 & 0 & 3 & - & - \\
\hline
\end{tabular}




\begin{tabular}{|c|c|c|c|c|c|c|c|c|c|c|c|c|c|c|c|c|c|c|c|c|c|c|c|c|c|c|c|}
\hline \# & Biodiesel & $\begin{array}{l}6: \\
0\end{array}$ & $8: 0$ & $10: 0$ & $12: 0$ & $14: 0$ & $14: 1$ & $15: 0$ & $16: 0$ & $16: 1$ & $17: 0$ & $17: 1$ & 18:0 & $18: 1$ & $18: 2$ & $18: 3$ & $20: 0$ & $20: 1$ & $20: 2$ & $20: 4$ & $22: 0$ & $22: 1$ & $24: 0$ & $24: 1$ & CFPP & $\mathrm{CP}$ & PP \\
\hline 11 & $\begin{array}{l}\text { Citrilus Colocynthis } \\
+ \text { Camelus } \\
\text { dromedaries Fat } \\
20: 80^{11}\end{array}$ & 0 & 0 & 0 & 0.3 & 6.2 & 1.1 & 1 & 24.8 & 7.4 & 1.7 & 0.7 & 12.9 & 26.9 & 15.1 & 0.8 & 0.2 & 0 & 0.1 & 0.1 & 0.1 & 0 & 0 & 0 & 13 & 8 & 2 \\
\hline 12 & $\begin{array}{l}\text { Citrilus Colocynthis } \\
+ \text { Camelus } \\
\text { dromedaries Fat } \\
40: 60^{11}\end{array}$ & 0 & 0 & 0 & 0.3 & 4.6 & 0.8 & 0.7 & 21.5 & 5.3 & 1.3 & 0.5 & 12.2 & 25.4 & 25.5 & 0.8 & 0.3 & 0.2 & 0.1 & 0.1 & 0.1 & 0 & 0.1 & 0 & 11 & 7 & 0 \\
\hline 13 & $\begin{array}{l}\text { Citrilus Colocynthis } \\
+ \text { Camelus } \\
\text { dromedaries Fat } \\
60: 40^{11}\end{array}$ & 0 & 0 & 0 & 0 & 3.6 & 0.6 & 0.6 & 18.7 & 3.8 & 1 & 0.4 & 10.9 & 23.2 & 35.7 & 0.7 & 0.3 & 0.3 & 0 & 0.1 & 0.1 & 0 & 0.1 & 0 & 9 & 5 & -2 \\
\hline 14 & $\begin{array}{l}\text { Citrilus Colocynthis } \\
+ \text { Camelus } \\
\text { dromedaries Fat } \\
80: 20^{11}\end{array}$ & 0 & 0 & 0 & 0.1 & 1.9 & 0.3 & 0.3 & 15.1 & 2 & 0.8 & 0.2 & 10.3 & 20.7 & 47 & 0.5 & 0.4 & 0.3 & 0 & 0 & 0.1 & 0 & 0.1 & 0 & 7 & 3 & -4 \\
\hline 15 & Citrilus Colocynthis ${ }^{11}$ & 0 & 0 & 0 & 0 & 0.1 & 0 & 0 & 9.4 & 0.1 & 0.1 & 0 & 7.5 & 16.2 & 65.6 & 0.3 & 0.4 & 0.2 & 0 & 0 & 0.1 & 0 & 0.1 & 0 & 4 & 0 & -7 \\
\hline 16 & Coconut $^{5}$ & 0 & 8 & 6.2 & 47.7 & 14.2 & 0 & 0 & 10.3 & 0 & 0 & 0 & 2.8 & 8.2 & 2.6 & 0 & 0 & 0 & 0 & 0 & 0 & 0 & 0 & 0 & -2 & 1 & -3 \\
\hline 17 & Coconut ${ }^{12}$ & $\begin{array}{l}0 . \\
4\end{array}$ & 7.5 & 5.8 & 45.6 & 18.4 & 0 & 0 & 9.5 & 0.1 & 0 & 0 & 2.9 & 7.6 & 2 & 0.1 & 0.1 & 0.1 & 0 & 0 & 0.1 & 0 & 0.1 & 0 & -2 & -4 & -5 \\
\hline 18 & Common Purslane $e^{13}$ & 0 & 0 & 0 & 0 & 0 & 0 & 0 & 16.4 & 0 & 0 & 0 & 3.6 & 11.8 & 34.1 & 32.4 & 0 & 0 & 0 & 0 & 0 & 0 & 0 & 0 & - & 3 & -6 \\
\hline 19 & Corn ${ }^{1}$ & 0 & 0 & 0 & 0 & 0 & 0 & 0 & 6.5 & 0.6 & 0 & 0 & 1.4 & 65.6 & 25.2 & 0.1 & 0.1 & 0.1 & 0 & 0 & 0 & 0.1 & 0.1 & 0 & -12 & - & - \\
\hline 20 & Cottonseed $^{14}$ & 0 & 0 & 0 & 0 & 0.8 & 0 & 0 & 24.4 & 0.4 & 0 & 0 & 2.2 & 17.2 & 55 & 0.3 & 0 & 0 & 0 & 0 & 0 & 0 & 0 & 0 & - & 9 & 2 \\
\hline 21 & $\begin{array}{l}\text { Croton } \\
\text { megalocarpus }{ }^{15}\end{array}$ & 0 & 0 & 0 & 0 & 0.1 & 0 & 0 & 6.5 & 0.1 & 0.1 & 0 & 3.8 & 11.6 & 72.7 & 3.9 & 0 & 0.9 & 0.2 & 0 & 0 & 0 & 0 & 0 & - & -4 & -9 \\
\hline
\end{tabular}




\begin{tabular}{|c|c|c|c|c|c|c|c|c|c|c|c|c|c|c|c|c|c|c|c|c|c|c|c|c|c|c|c|}
\hline$\#$ & Biodiesel & $\begin{array}{l}6: \\
0\end{array}$ & $8: 0$ & 10:0 & $12: 0$ & $14: 0$ & $14: 1$ & $15: 0$ & $16: 0$ & $16: 1$ & $17: 0$ & $17: 1$ & 18:0 & $18: 1$ & $18: 2$ & $18: 3$ & $20: 0$ & $20: 1$ & $20: 2$ & $20: 4$ & $22: 0$ & $22: 1$ & $24: 0$ & $24: 1$ & CFPP & $\mathrm{CP}$ & PP \\
\hline 22 & $\begin{array}{l}\text { Cynara } \\
\text { Cardunculus }{ }^{16}\end{array}$ & 0 & 0 & 0 & 0 & 0 & 0 & 0 & 7.6 & 0 & 0 & 0 & 0 & 25.8 & 41.7 & 3.8 & 0 & 0 & 0 & 0 & 0 & 10.2 & 0 & 0 & -3 & - & - \\
\hline 23 & $\begin{array}{l}\text { Dairy Washed Milk } \\
\text { Scum }^{17}\end{array}$ & $\begin{array}{l}0 . \\
6\end{array}$ & 0.9 & 2.3 & 3.3 & 12.2 & 0 & 0 & 37 & 0 & 0 & 0 & 12.7 & 26.9 & 0.4 & 0 & 0 & 0 & 0 & 0.9 & 0 & 0 & 0 & 0 & 17 & 17 & 10 \\
\hline 24 & Flaxseed $^{18}$ & 0 & 0 & 0 & 0 & 0 & 0 & 0 & 5.7 & 0 & 0 & 0 & 5.6 & 20.7 & 15.9 & 51.7 & 0.2 & 0 & 0 & 0 & 0.2 & 0 & 0 & 0 & - & -4 & -9 \\
\hline 25 & Grape $^{1}$ & 0 & 0 & 0 & 0 & 0.1 & 0 & 0 & 6.9 & 0.1 & 0 & 0 & 4 & 19 & 69.1 & 0.3 & 0.3 & 0 & 0 & 0 & 0 & 0 & 0 & 0 & -6 & - & - \\
\hline 26 & $\begin{array}{l}\text { High Oleic } \\
\text { Sunflower }{ }^{1}\end{array}$ & 0 & 0 & 0 & 0 & 0 & 0 & 0 & 4.6 & 0.1 & 0 & 0 & 3.4 & 62.8 & 27.5 & 0.1 & 0.3 & 0 & 0 & 0 & 0.7 & 0 & 0.3 & 0 & -6 & - & - \\
\hline 27 & Hazelnut ${ }^{19}$ & 0 & 0 & 0 & 0 & 0 & 0 & 0 & 5.1 & 0.4 & 0 & 0 & 2.1 & 78.3 & 13.1 & 0.2 & 0.2 & 0.3 & 0 & 0 & 0 & 0 & 0 & 0 & -12.7 & -9 & -13 \\
\hline 28 & $\begin{array}{l}\text { High Acid Methyl } \\
\text { Esther }^{20}\end{array}$ & 0 & 0 & 0 & 0 & 0.1 & 0 & 0 & 16.8 & 0.1 & 0 & 0 & 4.3 & 13.9 & 55.8 & 7.4 & 0.3 & 0 & 0 & 0 & 0 & 0 & 0 & 0 & 1 & 2 & 3 \\
\hline 29 & Sunflowerseed ${ }^{20}$ & 0 & 0 & 0 & 0 & 0 & 0 & 0 & 4.5 & 0 & 0 & 0 & 4 & 82 & 8 & 0.2 & 0.3 & 0 & 0 & 0 & 0 & 0 & 0 & 0 & - & - & -5 \\
\hline 30 & Jatropha curcas ${ }^{6}$ & 0 & 0 & 0 & 0 & 0.1 & 0 & 0 & 17.7 & 0.8 & 0 & 0 & 6.4 & 41.8 & 32.9 & 0.2 & 0.1 & 0 & 0 & 0 & 0 & 0 & 0 & 0 & - & 3 & 2 \\
\hline 31 & Jatropha $^{21}$ & 0 & 0 & 0 & 0 & 0 & 0 & 0 & 14.2 & 1.4 & 0 & 0 & 6.9 & 43.1 & 34.4 & 0 & 0 & 0 & 0 & 0 & 0 & 0 & 0 & 0 & - & 4 & -3 \\
\hline 32 & Jatropha $^{22}$ & 0 & 0 & 0 & 0 & 0.2 & 0 & 0 & 14.8 & 0.6 & 0 & 0 & 7.2 & 41.2 & 35.4 & 0.2 & 0 & 0.1 & 0 & 0 & 0 & 0 & 0 & 0 & -6 & - & - \\
\hline 33 & Jatropha $^{10}$ & 0 & 0 & 0 & 0 & 0.1 & 0 & 0 & 14 & 0.7 & 0.1 & 0.1 & 6.5 & 43.3 & 35 & 0.2 & 0 & 0 & 0 & 0 & 0 & 0 & 0 & 0 & 0 & - & - \\
\hline 34 & Jatropha $^{10}$ & 0 & 0 & 0 & 0 & 0 & 0 & 0 & 14.7 & 0.8 & 0.1 & 0 & 6.6 & 41.4 & 36.1 & 0.1 & 0.1 & 0 & 0 & 0 & 0 & 0 & 0 & 0 & 1 & - & - \\
\hline 35 & Linseed $^{23}$ & 0 & 0 & 0 & 0 & 0 & 0 & 0 & 6.7 & 0 & 0 & 0 & 3.7 & 21.7 & 15.8 & 52.1 & 0 & 0 & 0 & 0 & 0 & 0 & 0 & 0 & - & $\begin{array}{c}- \\
5.15\end{array}$ & - \\
\hline 36 & Mahua $^{24}$ & 0 & 0 & 0 & 0 & 0.1 & 0 & 0 & 21.5 & 0 & 0 & 0 & 19 & 39.1 & 19.6 & 0.2 & 0.6 & 0 & 0 & 0 & 0 & 0 & 0 & 0 & 8 & 13 & 6 \\
\hline 37 & Mahua ${ }^{24}$ & 0 & 0 & 0 & 0 & 0 & 0 & 0 & 21.4 & 0 & 0 & 0 & 19 & 39 & 19.5 & 0.2 & 0.9 & 0 & 0 & 0 & 0 & 0 & 0 & 0 & - & 15 & 10 \\
\hline 38 & Moringa Peregrina $a^{25}$ & 0 & 0 & 0 & 0 & 0 & 0 & 0 & 9.1 & 2.7 & 0 & 0 & 4 & 71.1 & 4.2 & 0.5 & 2.4 & 1.9 & 0 & 0 & 3.1 & 0 & 1 & 0 & - & - & 11 \\
\hline
\end{tabular}




\begin{tabular}{|c|c|c|c|c|c|c|c|c|c|c|c|c|c|c|c|c|c|c|c|c|c|c|c|c|c|c|c|}
\hline \# & Biodiesel & $\begin{array}{l}\text { 6: } \\
0\end{array}$ & $8: 0$ & $10: 0$ & $12: 0$ & $14: 0$ & $14: 1$ & $15: 0$ & $16: 0$ & $16: 1$ & $17: 0$ & $17: 1$ & $18: 0$ & $18: 1$ & $18: 2$ & $18: 3$ & $20: 0$ & $20: 1$ & $20: 2$ & $20: 4$ & $22: 0$ & $22: 1$ & $24: 0$ & $24: 1$ & CFPP & $\mathrm{CP}$ & PP \\
\hline 39 & Olive $^{1}$ & 0 & 0 & 0 & 0 & 0 & 0 & 0 & 11.6 & 1 & 0 & 0 & 3.1 & 75 & 7.8 & 0.6 & 0.3 & 0 & 0 & 0 & 0.1 & 0 & 0.5 & 0 & -6 & - & - \\
\hline 40 & Olive $^{23}$ & 0 & 0 & 0 & 0 & 0 & 0 & 0 & 10.7 & 0 & 0 & 0 & 2.6 & 78.7 & 5.8 & 0.7 & 0 & 0 & 0 & 0 & 0 & 0 & 0 & 0 & - & 5.15 & - \\
\hline 41 & Palm 1 & 0 & 0 & 0 & 0.1 & 0.7 & 0 & 0 & 36.7 & 0.1 & 0 & 0 & 6.6 & 46.1 & 8.6 & 0.3 & 0.4 & 0.2 & 0 & 0 & 0.1 & 0 & 0.1 & 0 & 10 & - & - \\
\hline 42 & Palm ${ }^{21}$ & 0 & 0 & 0 & 0 & 0 & 0 & 0 & 40.3 & 0 & 0 & 0 & 4.1 & 43.4 & 12.2 & 0 & 0 & 0 & 0 & 0 & 0 & 0 & 0 & 0 & - & 16 & 12 \\
\hline 43 & Palm²6 & 0 & 0 & 0 & 0.2 & 1 & 0 & 0 & 35.7 & 0.4 & 0 & 0 & 3.7 & 44.7 & 13.2 & 0.3 & 0.4 & 0.2 & 0 & 0 & 0.1 & 0 & 0.1 & 0 & - & 12 & 12 \\
\hline 44 & Palm $^{6}$ & 0 & 0 & 0 & 0.3 & 1 & 0 & 0 & 38.1 & 0.2 & 0 & 0 & 4.1 & 44.2 & 11 & 0.3 & 0.4 & 0.2 & 0 & 0 & 0.1 & 0 & 0.1 & 0 & - & 13 & 15 \\
\hline 45 & Palm ${ }^{27}$ & 0 & 0 & 0 & 0.3 & 1.1 & 0 & 0 & 41.9 & 0.2 & 0 & 0 & 4.6 & 41.2 & 10.3 & 0.1 & 0.3 & 0 & 0 & 0 & 0 & 0 & 0 & 0 & - & - & 8 \\
\hline 46 & Palm $^{10}$ & 0 & 0 & 0 & 0 & 1.1 & 0 & 0 & 44.4 & 0.1 & 0.1 & 0 & 3.8 & 39.5 & 10.1 & 0.3 & 0.2 & 0.1 & 0 & 0 & 0 & 0 & 0 & 0 & 11 & - & - \\
\hline 47 & Palm $^{10}$ & 0 & 0 & 0 & 0 & 1 & 0 & 0 & 44.2 & 0.2 & 0.1 & 0 & 4.2 & 38.9 & 10.3 & 0.2 & 0.4 & 0.1 & 0 & 0 & 0 & 0 & 0 & 0 & 11 & - & - \\
\hline 48 & Palm $^{23}$ & 0 & 0 & 0 & 0 & 0 & 0 & 0 & 39.5 & 0 & 0 & 0 & 4.1 & 43.2 & 10.6 & 0.2 & 0 & 0 & 0 & 0 & 0 & 0 & 0 & 0 & - & 9.85 & - \\
\hline 49 & Peanut ${ }^{1}$ & 0 & 0 & 0 & 0 & 0.1 & 0 & 0 & 8 & 0 & 0 & 0 & 1.8 & 53.3 & 28.4 & 0.3 & 0.9 & 2.4 & 0 & 0 & 3 & 0 & 1.8 & 0 & 17 & - & - \\
\hline 50 & Peanut ${ }^{19}$ & 0 & 0 & 0 & 0 & 0 & 0 & 0 & 6.7 & 0 & 0 & 0 & 2.3 & 78.9 & 4.4 & 0 & 1.2 & 1.9 & 0 & 0 & 2.6 & 0 & 1.8 & 0 & 16 & 18 & 15 \\
\hline 51 & Peanut ${ }^{26}$ & 0 & 0 & 0 & 0 & 0 & 0 & 0 & 8.2 & 0 & 0 & 0 & 1.7 & 54.2 & 27.5 & 0.2 & 0.9 & 2.4 & 0 & 0 & 2.9 & 0.2 & 1.8 & 0 & - & 20 & 15 \\
\hline 52 & Penycress ${ }^{8}$ & 0 & 0 & 0 & 0 & 0 & 0 & 0 & 3.1 & 0.2 & 0 & 0 & 0.5 & 12.6 & 22.4 & 11.8 & 2.4 & 9.8 & 0 & 0 & 0.5 & 35 & 0 & 0 & -12.7 & 11.6 & -17 \\
\hline 53 & Pongamia ${ }^{21}$ & 0 & 0 & 0 & 0 & 0 & 0 & 0 & 9.8 & 0 & 0 & 0 & 6.2 & 72.2 & 11.8 & 0 & 0 & 0 & 0 & 0 & 0 & 0 & 0 & 0 & - & -1 & -6 \\
\hline 54 & Poppyseed ${ }^{28}$ & 0 & 0 & 0 & 0 & 0 & 0 & 0 & 9 & 0 & 0 & 0 & 2.2 & 13.7 & 74.1 & 1 & 0 & 0 & 0 & 0 & 0 & 0 & 0 & 0 & -7 & -8 & - \\
\hline 55 & Poppyseed ${ }^{29}$ & 0 & 0 & 0 & 0 & 0 & 0 & 0 & 9.4 & 0.2 & 0 & 0 & 3.1 & 16.6 & 68.6 & 0.8 & 0 & 0 & 0 & 0 & 0 & 0 & 0 & 0 & - & -5 & -13 \\
\hline 56 & $\begin{array}{l}\text { Rapeseed + } \\
\text { Sunflower }{ }^{30}\end{array}$ & 0 & 0 & 0 & 0 & 0.2 & 0 & 0 & 7.8 & 0.2 & 0 & 0 & 3.2 & 36.2 & 48.3 & 4.2 & 0 & 0 & 0 & 0 & 0 & 0 & 0 & 0 & -8 & -2 & - \\
\hline
\end{tabular}




\begin{tabular}{|c|c|c|c|c|c|c|c|c|c|c|c|c|c|c|c|c|c|c|c|c|c|c|c|c|c|c|c|}
\hline \# & Biodiesel & $\begin{array}{l}6: \\
0\end{array}$ & $8: 0$ & 10:0 & 12:0 & 14:0 & $14: 1$ & $15: 0$ & 16:0 & 16:1 & 17:0 & $17: 1$ & $18: 0$ & $18: 1$ & $18: 2$ & $18: 3$ & $20: 0$ & $20: 1$ & $20: 2$ & $20: 4$ & 22:0 & 22:1 & $24: 0$ & $24: 1$ & CFPP & $\mathrm{CP}$ & $\mathrm{PP}$ \\
\hline 57 & Rapeseed $^{1}$ & 0 & 0 & 0 & 0 & 0 & 0 & 0 & 4.9 & 0 & 0 & 0 & 1.6 & 33 & 20.4 & 7.9 & 0 & 9.3 & 0 & 0 & 0 & 23 & 0 & 0 & -10 & - & - \\
\hline 58 & Rapeseed $^{30}$ & 0 & 0 & 0 & 0 & 4.5 & 0 & 0 & 0 & 0 & 0 & 0 & 2.1 & 64.1 & 18.9 & 8.8 & 0.6 & 1 & 0 & 0 & 0 & 0 & 0 & 0 & -11 & -7 & - \\
\hline 59 & Rapeseed $^{30}$ & 0 & 0 & 0 & 0.1 & 0.1 & 0 & 0 & 5.4 & 0.3 & 0 & 0 & 1.9 & 63.3 & 19.6 & 6.7 & 0.5 & 1.2 & 0.1 & 0 & 0.3 & 0.4 & 0.2 & 0 & -8 & -5 & - \\
\hline 60 & Rapeseed $^{16}$ & 0 & 0 & 0 & 0 & 0 & 0 & 0 & 4.5 & 0 & 0 & 0 & 0 & 66.6 & 17.2 & 7.8 & 0 & 0 & 0 & 0 & 0 & 0 & 0 & 0 & -7 & - & - \\
\hline 61 & Rapeseed $^{31}$ & 0 & 0 & 0 & 0 & 0 & 0 & 0 & 3.5 & 0 & 0 & 0 & 0.9 & 64.4 & 22.3 & 8.2 & 0 & 0 & 0 & 0 & 0 & 0 & 0 & 0 & - & -4 & -12 \\
\hline 62 & Rapeseed $^{32}$ & 0 & 0 & 0 & 0 & 0 & 0 & 0 & 8.2 & 0.3 & 0 & 0 & 2.9 & 53.8 & 23.4 & 9.8 & 1 & 0 & 0 & 0 & 0.5 & 0 & 0 & 0 & -11 & -8 & -15 \\
\hline 63 & Rapeseed $^{23}$ & 0 & 0 & 0 & 0 & 0 & 0 & 0 & 4.3 & 0 & 0 & 0 & 1.9 & 61.5 & 20.2 & 18.3 & 0 & 0 & 0 & 0 & 0 & 0 & 0 & 0 & - & 6.15 & - \\
\hline 64 & Rapeseed $^{26}$ & 0 & 0 & 0 & 0 & 0.1 & 0 & 0 & 4.6 & 0.3 & 0 & 0 & 1.8 & 62.4 & 19.3 & 8.4 & 0.6 & 1.4 & 0 & 0 & 0.3 & 0.2 & 0.2 & 0.4 & - & -3 & -9 \\
\hline 65 & Rapeseed $^{10}$ & 0 & 0 & 0 & 0 & 0.1 & 0 & 0 & 4.6 & 0.3 & 0.1 & 0.1 & 1.5 & 63.9 & 20.4 & 7 & 0.7 & 1.3 & 0 & 0 & 0 & 0 & 0 & 0 & -14 & - & - \\
\hline 66 & Rubberseed ${ }^{31}$ & 0 & 0 & 0 & 0 & 0 & 0 & 0 & 10.2 & 0 & 0 & 0 & 8.7 & 24.6 & 39.6 & 16.3 & 0 & 0 & 0 & 0 & 0 & 0 & 0 & 0 & - & 4 & -8 \\
\hline 67 & Safflower ${ }^{23}$ & 0 & 0 & 0 & 0 & 0 & 0 & 0 & 6.4 & 0 & 0 & 0 & 2.2 & 13.9 & 76 & 0.2 & 0 & 0 & 0 & 0 & 0 & 0 & 0 & 0 & - & 6.15 & - \\
\hline 68 & Saflower ${ }^{32}$ & 0 & 0 & 0 & 0 & 0 & 0 & 0 & 11.1 & 0 & 0 & 0 & 4.4 & 12.8 & 69.7 & 0.5 & 0.8 & 0 & 0 & 0 & 0.6 & 0 & 0.3 & 0 & -9 & - & -13 \\
\hline 69 & Soybean ${ }^{1}$ & 0 & 0 & 0 & 0 & 0 & 0 & 0 & 11.3 & 0.1 & 0 & 0 & 3.6 & 24.9 & 53 & 6.1 & 0.3 & 0.3 & 0 & 0 & 0 & 0.3 & 0.1 & 0 & -5 & - & - \\
\hline 70 & Soybean ${ }^{20}$ & 0 & 0 & 0 & 0 & 0 & 0 & 0 & 11.2 & 0 & 0 & 0 & 4.3 & 22.6 & 51.2 & 9.9 & 0.3 & 0 & 0 & 0 & 0 & 0 & 0 & 0 & -4 & 0.3 & -2 \\
\hline 71 & Soybean $^{20}$ & 0 & 0 & 0 & 0.3 & 0.2 & 0 & 0 & 15.2 & 0 & 0 & 0 & 4 & 22.2 & 50.2 & 6.5 & 0.2 & 0 & 0 & 0 & 0 & 0 & 0 & 0 & -4 & -1.1 & -1.6 \\
\hline 72 & Soybean $^{20}$ & 0 & 0 & 0 & 0 & 0 & 0 & 0 & 12.5 & 0 & 0 & 0 & 4.3 & 24.2 & 51.5 & 7.6 & 0 & 0 & 0 & 0 & 0 & 0 & 0 & 0 & -4 & 1.4 & -1.3 \\
\hline 73 & Soybean ${ }^{20}$ & 0 & 0 & 0 & 0.4 & 3 & 0 & 0 & 12.9 & 0 & 0 & 0 & 5.5 & 24.5 & 45.6 & 8 & 0 & 0 & 0 & 0 & 0 & 0 & 0 & 0 & -4 & 6.4 & 0 \\
\hline 74 & Soybean ${ }^{20}$ & 0 & 0 & 0 & 0 & 0 & 0 & & 10.2 & 0 & 0 & 0 & 4.6 & 24.2 & 53.3 & 7.2 & 0 & 0 & 0 & 0 & 0 & 0 & 0 & 0 & -4 & 1.5 & 0 \\
\hline 75 & Soybean ${ }^{20}$ & 0 & 0 & 0 & 0 & 0 & 0 & 0 & 12.5 & 0 & 0 & 0 & 4.3 & 24.2 & 51.5 & 7.6 & 0 & 0 & 0 & 0 & 0 & 0 & 0 & 0 & -2.4 & 1.3 & -0.8 \\
\hline
\end{tabular}




\begin{tabular}{|c|c|c|c|c|c|c|c|c|c|c|c|c|c|c|c|c|c|c|c|c|c|c|c|c|c|c|c|}
\hline \# & Biodiesel & $\begin{array}{l}\text { 6: } \\
0\end{array}$ & $8: 0$ & 10:0 & $12: 0$ & $14: 0$ & $14: 1$ & $15: 0$ & 16:0 & $16: 1$ & 17:0 & $17: 1$ & $18: 0$ & $18: 1$ & $18: 2$ & $18: 3$ & $20: 0$ & $20: 1$ & $20: 2$ & $20: 4$ & $22: 0$ & 22:1 & $24: 0$ & $24: 1$ & CFPP & $\mathrm{CP}$ & PP \\
\hline 76 & Soybean ${ }^{20}$ & 0 & 0 & 0 & 0 & 0 & 0 & 0 & 11.7 & 0 & 0 & 0 & 4.3 & 24.8 & 50.8 & 8.4 & 0.1 & 0 & 0 & 0 & 0 & 0 & 0 & 0 & - & 3.2 & -2 \\
\hline 77 & Soybean ${ }^{4}$ & 0 & 0 & 0 & 0 & 0.1 & 0 & 0 & 10.9 & 0 & 0 & 0 & 3.2 & 26.7 & 51.8 & 5.6 & 0.8 & 0 & 0 & 0 & 1 & 0 & 0 & 0 & -4 & - & - \\
\hline 78 & Soybean ${ }^{16}$ & 0 & 0 & 0 & 0 & 0 & 0 & 0 & 10.6 & 0 & 0 & 0 & 0 & 25.1 & 52.1 & 5.9 & 0 & 0 & 0 & 0 & 0 & 0.4 & 0 & 0 & -4 & - & - \\
\hline 79 & Soybean $^{8}$ & 0 & 0 & 0 & 0 & 0 & 0 & 0 & 10.5 & 0 & 0 & 0 & 4.7 & 22.7 & 53.4 & 8.2 & 0 & 0 & 0 & 0 & 0 & 0 & 0 & 0 & -3 & 1.5 & 0 \\
\hline 80 & Soybean ${ }^{31}$ & 0 & 0 & 0 & 0 & 0 & 0 & 0 & 11.8 & 0 & 0 & 0 & 3.2 & 23.3 & 55.5 & 6.3 & 0 & 0 & 0 & 0 & 0 & 0 & 0 & 0 & - & -2 & -3 \\
\hline 81 & Soybean ${ }^{32}$ & 0 & 0 & 0 & 0 & 0 & 0 & 0 & 16.3 & 0 & 0 & 0 & 6.7 & 22.7 & 44.1 & 9 & 0.6 & 0 & 0 & 0 & 0.6 & 0 & 0 & 0 & -2 & 1 & 0 \\
\hline 82 & Soybean ${ }^{23}$ & 0 & 0 & 0 & 0 & 0 & 0 & 0 & 10.7 & 0 & 0 & 0 & 3.2 & 25 & 53.3 & 5.4 & 0 & 0 & 0 & 0 & 0 & 0 & 0 & 0 & - & 1.15 & - \\
\hline 83 & Soybean ${ }^{26}$ & 0 & 0 & 0 & 0.1 & 0.1 & 0 & 0 & 10.8 & 0.2 & 0 & 0 & 3.6 & 24.9 & 52.7 & 6 & 0.4 & 0.4 & 0 & 0 & 0.5 & 0.1 & 0.2 & 0 & - & 2 & 0 \\
\hline 84 & Soybean ${ }^{10}$ & 0 & 0 & 0 & 0 & 0.1 & 0 & 0 & 10.4 & 0.1 & 0.1 & 0 & 4.2 & 23.9 & 54.8 & 6.1 & 0.2 & 0.1 & 0 & 0 & 0 & 0 & 0 & 0 & -4 & - & - \\
\hline 85 & $\begin{array}{l}\text { Stauntonia } \\
\text { Chinensis }{ }^{33}\end{array}$ & 0 & 0 & 0 & 0 & 0 & 0 & 0 & 6.9 & 0.2 & 0 & 0 & 1.2 & 80 & 8.3 & 0.1 & 1.7 & 0.5 & 0 & 0 & 0 & 0 & 0 & 0 & -9 & - & - \\
\hline 86 & Sunflower ${ }^{1}$ & 0 & 0 & 0 & 0 & 0 & 0 & 0 & 6.2 & 0.1 & 0 & 0 & 3.7 & 25.2 & 63.1 & 0.2 & 0.3 & 0.2 & 0 & 0 & 0.7 & 0.1 & 0.2 & 0 & -3 & - & - \\
\hline 87 & Sunflower ${ }^{23}$ & 0 & 0 & 0 & 0 & 0 & 0 & 0 & 6.1 & 0 & 0 & 0 & 4.2 & 24 & 63.5 & 0.4 & 0 & 0 & 0 & 0 & 0 & 0 & 0 & 0 & - & 0.85 & - \\
\hline 88 & Sunflower ${ }^{26}$ & 0 & 0 & 0 & 0 & 0.1 & 0 & 0 & 6.6 & 0.2 & 0 & 0 & 3.4 & 28.6 & 59.5 & 0.1 & 0.3 & 0.2 & 0 & 0 & 0.8 & 0.1 & 0.3 & 0 & - & 4 & -6 \\
\hline 89 & Tallow $^{10}$ & 0 & 0 & 0 & 0 & 1.8 & 0.2 & 0 & 23.7 & 2.9 & 0.6 & 0.5 & 13.8 & 42.8 & 10.2 & 0.9 & 0.3 & 0.9 & 0 & 0 & 0 & 0 & 0 & 0 & 10 & - & - \\
\hline 90 & Tallow ${ }^{10}$ & 0 & 0 & 0 & 0 & 3 & 1.1 & 0 & 23.8 & 5.5 & 1.2 & 0.7 & 22.3 & 37.9 & 2.3 & 0.3 & 0.5 & 0.1 & 0 & 0 & & 0 & 0 & 0 & 14 & - & - \\
\hline 91 & Used cooking oil20 & 0 & 0 & 0 & 0 & 0.6 & 0 & 0 & 15.3 & 0.9 & 0 & 0 & 8.1 & 49 & 24 & 2.2 & 0 & 0 & 0 & 0 & 0 & 0 & 0 & 0 & 2 & 8.4 & 3.3 \\
\hline 92 & Walnut ${ }^{19}$ & 0 & 0 & 0 & 0 & 0 & 0 & 0 & 7.2 & 0 & 0 & 0 & 2.6 & 15.9 & 60.7 & 12.8 & 0 & 0.2 & 0 & 0 & 0 & 0 & 0 & 0 & -9 & -6 & -10 \\
\hline 93 & Waste cooking oil ${ }^{34}$ & 0 & 0 & 0 & 0 & 1 & 0 & 0 & 20.1 & 0 & 0 & 0 & 14.9 & 21.7 & 35.4 & 5.1 & 0 & 0 & 0 & 0 & 0 & 0 & 0 & 0 & 2 & - & -4 \\
\hline 94 & Waste cooking oil ${ }^{35}$ & 0 & 0 & 0 & 0 & 0.3 & 0 & 0 & 7.7 & 0.5 & 0 & 0 & 3.5 & 32.3 & 53.3 & 0.1 & 0.3 & 0.3 & 0 & 0 & 1.3 & 0 & 0.5 & 0 & -2 & - & -3 \\
\hline
\end{tabular}




\begin{tabular}{|c|c|c|c|c|c|c|c|c|c|c|c|c|c|c|c|c|c|c|c|c|c|c|c|c|c|c|c|}
\hline$\#$ & Biodiesel & $\begin{array}{l}6: \\
0\end{array}$ & $8: 0$ & 10:0 & $12: 0$ & $14: 0$ & $14: 1$ & $15: 0$ & $16: 0$ & $16: 1$ & $17: 0$ & $17: 1$ & $18: 0$ & $18: 1$ & $18: 2$ & $18: 3$ & $20: 0$ & $20: 1$ & $20: 2$ & $20: 4$ & $22: 0$ & $22: 1$ & $24: 0$ & $24: 1$ & CFPP & $\mathrm{CP}$ & $\mathrm{PP}$ \\
\hline 95 & Waste cooking oil ${ }^{8}$ & 0 & 0 & 0 & 0 & 0 & 0 & 0 & 10.9 & 0.6 & 0 & 0 & 4 & 38.1 & 40.5 & 4.7 & 0.4 & 0 & 0 & 0 & 0 & 0 & 0 & 0 & -4 & -0.6 & -2 \\
\hline 96 & Waste cooking oil36 & 0 & 0 & 0 & 0 & 0.6 & 0 & 0 & 36.2 & 0 & 0 & 0 & 4.6 & 45 & 13.2 & 0 & 0.5 & 0 & 0 & 0 & 0 & 0 & 0 & 0 & - & 10 & 12 \\
\hline 97 & Waste Frying $\mathrm{Oi}^{37}$ & 0 & 0.1 & 0.3 & 1.2 & 0.1 & 0 & 0 & 37.3 & 0.1 & 0 & 0 & 4 & 40.4 & 17.8 & 0.2 & 0 & 0 & 0 & 0 & 0 & 0 & 0 & 0 & 7 & 9.5 & 5 \\
\hline 98 & Yellow Grease ${ }^{8}$ & 0 & 0 & 0 & 0 & 0.5 & 0 & 0 & 6.6 & 0.5 & 0 & 0 & 4.3 & 66.1 & 17.2 & 2 & 0.4 & 0.5 & 0 & 0 & 0.5 & 0 & 0 & 0 & 0.3 & 2.5 & -5 \\
\hline 99 & Canola $^{38}$ & 0 & 0 & 0 & 0 & 0.2 & 0 & 0 & 4.4 & 0.3 & 0 & 0 & 2 & 59.4 & 21.2 & 10.4 & 0.5 & 0.8 & 0 & 0 & 0 & 0.8 & 0 & 0 & -4 & - & -6 \\
\hline 100 & Coconut ${ }^{38}$ & 0 & 0 & 0 & 0 & 1 & 0 & 0 & 17.8 & 0 & 0 & 0 & 21.8 & 48 & 9.3 & 0 & 0 & 0 & 0 & 0 & 0 & 0 & 0 & 0 & - & 12 & - \\
\hline 101 & Coconut ${ }^{39}$ & 0 & 0.4 & 1.3 & 22 & 15.2 & 0 & 0 & 17.3 & 0 & 0 & 0 & 2.6 & 29.4 & 13.3 & 0 & 0 & 0 & 0 & 0 & 0 & 0 & 0 & 0 & - & 4 & - \\
\hline 102 & Corn $^{40}$ & 0 & 0 & 0 & 0 & 0 & 0 & 0 & 10.3 & 0 & 0 & 0 & 2.3 & 31 & 54.5 & 0.8 & 0.4 & 0.1 & 0 & 0 & 0.2 & 0 & 0.2 & 0 & -5 & - & - \\
\hline 103 & Corn ${ }^{38}$ & 0 & 0 & 0 & 0 & 0.2 & 0 & 0 & 12.1 & 0.1 & 0 & 0 & 2.3 & 30.9 & 53.3 & 1.1 & 0 & 0 & 0 & 0 & 0 & 0 & 0 & 0 & -5 & - & -6.5 \\
\hline 104 & Fleshing $^{40}$ & 0 & 0 & 0 & 0 & 3.1 & 1.4 & 0.4 & 26.2 & 6.1 & 0.9 & 0.9 & 11.3 & 44.8 & 3.5 & 0.3 & 0 & 0.4 & 0 & 0 & 0 & 0 & 0 & 0 & 10 & - & - \\
\hline 105 & Jatropha Curcas ${ }^{41}$ & 0 & 0 & 0 & 0.1 & 0.1 & 0 & 0 & 14.6 & 0.6 & 0 & 0 & 7.6 & 44.6 & 31.9 & 0.3 & 0.3 & 0 & 0 & 0 & 0 & 0 & 0 & 0 & - & 3 & - \\
\hline 106 & Jatropha $^{39}$ & 0 & 0 & 0 & 0 & 0.5 & 0 & 0 & 19.5 & 0 & 0 & 0 & 3 & 38.1 & 34.3 & 0.6 & 2 & 0 & 0 & 0 & 0 & 0 & 0 & 0 & - & 4 & - \\
\hline 107 & Mahua $^{39}$ & 0 & 0 & 0 & 0 & 0.2 & 0 & 0 & 13.3 & 0 & 0 & 0 & 7.1 & 41.8 & 35.8 & 0.3 & 0.3 & 0 & 0 & 0 & 0 & 0 & 0 & 0 & - & 3 & - \\
\hline 108 & Olive $^{38}$ & 0 & 0 & 0 & 0 & 0 & 0 & 0 & 13.6 & 1.7 & 0 & 0 & 2.7 & 68.2 & 11.5 & 0.9 & 0.5 & 0.2 & 0 & 0 & 0.1 & 0 & 0.6 & 0 & -2 & - & -4 \\
\hline 109 & Palm + Soybean ${ }^{42}$ & 0 & 0 & 0 & 0 & 0.2 & 0 & 0 & 15.6 & 0.1 & 0.1 & 0 & 3.8 & 26.2 & 47.3 & 5.4 & 0.4 & 0.3 & 0 & 0 & 0.4 & 0 & 0.2 & 0 & -1 & 2.1 & - \\
\hline 110 & Palm 43 & 0 & 0 & 0 & 0 & 2.3 & 0 & 0 & 44.2 & 0 & 0 & 0 & 3.6 & 41.1 & 8.4 & 0.1 & 0.1 & 0.2 & 0 & 0 & 0 & 0 & 0 & 0 & 16 & 18 & 13 \\
\hline 111 & Palm 39 & 0 & 0.1 & 0 & 0.3 & 0.7 & 0 & 0 & 36.8 & 0 & 0 & 0 & 0 & 46.5 & 13.7 & 0.5 & 0.1 & 0 & 0 & 0 & 0.1 & 0 & 0.1 & 0 & - & 10 & - \\
\hline 112 & Rapeseed $^{44}$ & 0 & 0 & 0 & 0 & 0 & 0 & 0 & 3 & 0 & 0 & 0 & 1 & 64 & 22 & 8 & 0 & 0 & 0 & 0 & 0 & 0 & 0 & 0 & - & - & -13 \\
\hline 113 & Rice bran ${ }^{39}$ & 0 & 0 & 0 & 0 & 0 & 0 & 0 & 16.4 & 0 & 0 & 0 & 3.6 & 11.8 & 34.1 & 32.4 & 0 & 0 & 0 & 0 & 0 & 0 & 0 & 0 & - & 3 & -6 \\
\hline 114 & Soybean ${ }^{45}$ & 0 & 0 & 0 & 0 & 0 & 0 & 0 & 11.4 & 0 & 0 & 0 & 4.1 & 17.6 & 59 & 8 & 0 & 0 & 0 & 0 & 0 & 0 & 0 & 0 & -5 & - & - \\
\hline
\end{tabular}




\begin{tabular}{|c|c|c|c|c|c|c|c|c|c|c|c|c|c|c|c|c|c|c|c|c|c|c|c|c|c|c|c|}
\hline \# & Biodiesel & $\begin{array}{l}6: \\
0\end{array}$ & $8: 0$ & 10:0 & $12: 0$ & $14: 0$ & $14: 1$ & $15: 0$ & $16: 0$ & $16: 1$ & $17: 0$ & $17: 1$ & $18: 0$ & $18: 1$ & $18: 2$ & $18: 3$ & $20: 0$ & $20: 1$ & $20: 2$ & $20: 4$ & 22:0 & $22: 1$ & $24: 0$ & $24: 1$ & CFPP & $\mathrm{CP}$ & PP \\
\hline 115 & Soybean ${ }^{38}$ & 0 & 0 & 0 & 0.1 & 0.2 & 0 & 0 & 10.5 & 0 & 0 & 0 & 3.8 & 23.7 & 54.5 & 6.3 & 0.3 & 0 & 0 & 0 & 0.3 & 0.3 & 0 & 0 & -7 & - & -9 \\
\hline 116 & Soybean ${ }^{44}$ & 0 & 0 & 0 & 0 & 0 & 0 & 0 & 12 & 0 & 0 & 0 & 3 & 23 & 55 & 6 & 0 & 0 & 0 & 0 & 0 & 0 & 0 & 0 & - & - & -2 \\
\hline 117 & Stone Fruit Kernel ${ }^{46}$ & 0 & 0 & 0 & 0 & 0 & 0 & 0 & 5.9 & 0 & 0 & 0 & 2.5 & 63.8 & 25.3 & 0.5 & 0 & 0 & 0 & 0 & 0.7 & 0 & 0 & 0 & - & -4 & -8 \\
\hline 118 & Sunflower ${ }^{39}$ & 0 & 0 & 0 & 0 & 0.1 & 0 & 0 & 6.3 & 0 & 0 & 0 & 3.8 & 33.5 & 54.7 & 0 & 0.2 & 0 & 0 & 0 & 0.8 & 0 & 0.3 & 0 & - & -2 & - \\
\hline 119 & Sunflower ${ }^{44}$ & 0 & 0 & 0 & 0 & 0 & 0 & 0 & 6 & 0 & 0 & 0 & 3 & 17 & 74 & 0 & 0 & 0 & 0 & 0 & 0 & 0 & 0 & 0 & - & - & -5 \\
\hline 120 & Yellow Grease ${ }^{40}$ & 0 & 0 & 0 & 0 & 0.5 & 0 & 0 & 19.4 & 3.6 & 0.4 & 0 & 6.1 & 32.2 & 32.8 & 2.9 & 0 & 0 & 0 & 0.6 & 0.3 & 0 & 0 & 0 & 3 & - & - \\
\hline
\end{tabular}

Data in gray were used only for the validation step 


\section{References}

(1) Ramos, M. J.; Fernández, C. M.; Casas, A.; Rodríguez, L.; Pérez, Á. Influence of Fatty Acid Composition of Raw Materials on Biodiesel Properties. Bioresour. Technol. 2009, 100 (1), 261-268. https://doi.org/10.1016/j.biortech.2008.06.039.

(2) Palash, S. M.; Masjuki, H. H.; Kalam, M. A.; Atabani, A. E.; Fattah, I. M. R.; Sanjid, A. Biodiesel Production, Characterization, Diesel Engine Performance, and Emission Characteristics of Methyl Esters from Aphanamixis Polystachya Oil of Bangladesh. ENERGY Convers. Manag. $\quad$ 2015, $\quad$ 91, 149-157. https://doi.org/10.1016/j.enconman.2014.12.009.

(3) Folayan, A. J.; Anawe, P. A. L. Synthesis and Characterization of Argania Spinosa (Argan Oil) Biodiesel by Sodium Hydroxide Catalyzed Transesterification Reaction as Alternative for Petro-Diesel in Direct Injection, Compression Ignition Engines. Heliyon 2019, 5 (9), e02427. https://doi.org/10.1016/j.heliyon.2019.e02427.

(4) de Freitas, O. N.; Cardoso Rial, R.; Fontoura Cavalheiro, L.; dos Santos Barbosa, J. M.; Domingues Nazário, C. E.; Viana, L. H. Evaluation of the Oxidative Stability and Cold Filter Plugging Point of Soybean Methyl Biodiesel/Bovine Tallow Methyl Biodiesel

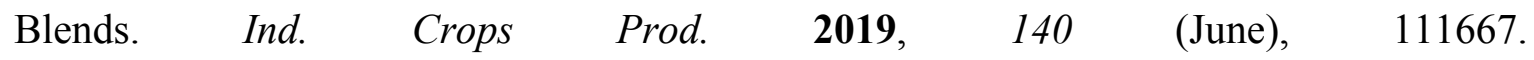
https://doi.org/10.1016/j.indcrop.2019.111667.

(5) Islam, M. M.; Hassan, M. H.; Kalam, M. A.; Zulkifli, N. W. binti M.; Habibullah, M.; Hossain, M. M. Improvement of Cold Flow Properties of Cocos Nucifera and Calophyllum Inophyllum Biodiesel Blends Using Polymethyl Acrylate Additive. J. Clean. Prod. 2016, 
137, 322-329. https://doi.org/10.1016/j.jclepro.2016.07.080.

(6) Amin, R.; Abedin, J.; Rahman, S. M. A.; Bin, M.; Hassan, H.; Alabdulkarem, A.; Kalam, A.; Islam, S. Impact of Fatty Acid Composition and Physicochemical Properties of Jatropha and Alexandrian Laurel Biodiesel Blends: An Analysis of Performance and Emission Characteristics. J. Clean. Prod. 2016. https://doi.org/10.1016/j.jclepro.2016.06.017.

(7) Fröhlich, A.; Rice, B. Evaluation of Camelina Sativa Oil as a Feedstock for Biodiesel Production. Ind. Crops Prod. 2005, $21 \quad$ (1), 25-31. https://doi.org/10.1016/j.indcrop.2003.12.004.

(8) Moser, B. R. Impact of Fatty Ester Composition on Low Temperature Properties of Biodiesel-Petroleum Diesel Blends. Fuel 2014, 115 (July), 500-506. https://doi.org/10.1016/j.fuel.2013.07.075.

(9) Öztürk, E. Performance, Emissions, Combustion and Injection Characteristics of a Diesel Engine Fuelled with Canola Oil-Hazelnut Soapstock Biodiesel Mixture. Fuel Process. Technol. 2015, 129, 183-191. https://doi.org/10.1016/j.fuproc.2014.09.016.

(10) Echim, C.; Maes, J.; Greyt, W. De. Improvement of Cold Filter Plugging Point of Biodiesel from Alternative Feedstocks. Fuel 2012, 93, 642-648. https://doi.org/10.1016/j.fuel.2011.11.036.

(11) Sbihi, H. M.; Nehdi, I. A.; Mokbli, S.; Romdhani-Younes, M.; Al-Resayes, S. I. Study of Oxidative Stability and Cold Flow Properties of Citrillus Colocynthis Oil and Camelus Dromedaries Fat Biodiesel Blends. Ind. Crops Prod. 2018, 122 (May 2018), 133-141. https://doi.org/10.1016/j.indcrop.2018.05.071. 
(12) Monirul, I. M.; Kalam, M. A.; Masjuki, H. H.; Zulkifli, N. W. M.; Shahir, S. A.; Mosarof, M. H.; Ruhul, A. M. Influence of Poly(Methyl Acrylate) Additive on Cold Flow Properties of Coconut Biodiesel Blends and Exhaust Gas Emissions. Renew. Energy 2017, 101, 702712. https://doi.org/10.1016/j.renene.2016.09.020.

(13) Hoseini, S. S.; Naja, G.; Sadeghi, A. Industrial Crops \& Products Chemical Characterization of Oil and Biodiesel from Common Purslane ( Portulaca) Seed as Novel Weed Plant Feedstock. 2019, 140 (July). https://doi.org/10.1016/j.indcrop.2019.111582.

(14) Shankar, A. A.; Pentapati, P. R.; Prasad, R. K. Biodiesel Synthesis from Cottonseed Oil Using Homogeneous Alkali Catalyst and Using Heterogeneous Multi Walled Carbon Nanotubes: Characterization and Blending Studies. Egypt. J. Pet. 2017, 26 (1), 125-133. https://doi.org/10.1016/j.ejpe.2016.04.001.

(15) Kafuku, G.; Kee, M.; Kansedo, J.; Teong, K.; Mbarawa, M. Bioresource Technology Croton Megalocarpus Oil : A Feasible Non-Edible Oil Source for Biodiesel Production. Bioresour. Technol. 2010, 101 (18), 7000-7004. https://doi.org/10.1016/j.biortech.2010.03.144.

(16) Martínez, G.; Sánchez, N.; Encinar, J. M.; González, J. F. Fuel Properties of Biodiesel from Vegetable Oils and Oil Mixtures. Influence of Methyl Esters Distribution. Biomass and Bioenergy 2014, 63, 22-32. https://doi.org/10.1016/j.biombioe.2014.01.034.

(17) Srikanth, H. V.; Venkatesh, J.; Godiganur, S.; Venkateswaran, S.; Manne, B. Bio-Based Diluents Improve Cold Flow Properties of Dairy Washed Milk-Scum Biodiesel. Renew. Energy 2017, 111, 168-174. https://doi.org/10.1016/j.renene.2017.03.092.

(18) Ahmad, T.; Danish, M.; Kale, P.; Geremew, B.; Adeloju, S. B.; Nizami, M.; Ayoub, M. 
Optimization of Process Variables for Biodiesel Production by Transesteri Fi Cation of Fl Axseed Oil and Produced Biodiesel Characterizations. Renew. Energy 2019, 139, 12721280. https://doi.org/10.1016/j.renene.2019.03.036.

(19) Moser, B. R. Preparation of Fatty Acid Methyl Esters from Hazelnut, High-Oleic Peanut and Walnut Oils and Evaluation as Biodiesel. Fuel 2012, 92 (1), 231-238. https://doi.org/10.1016/j.fuel.2011.08.005.

(20) Dunn, R. O. Cold Flow Properties of Biodiesel by Automatic and Manual Analysis Methods. J. ASTM Int. 2010, 7 (4). https://doi.org/10.1520/JAI102618.

(21) Sarin, A.; Arora, R.; Singh, N. P.; Sarin, R.; Malhotra, R. K.; Kundu, K. Effect of Blends of Palm-Jatropha-Pongamia Biodiesels on Cloud Point and Pour Point. Energy 2009, 34 (11), 2016-2021. https://doi.org/10.1016/j.energy.2009.08.017.

(22) Kivevele, T. T.; Huan, Z. Effects of Antioxidants on the Cetane Number, Viscosity, Oxidation Stability, and Thermal Properties of Biodiesel Produced from Nonedible Oils. Energy Technol. 2013, 1 (9), 537-543. https://doi.org/10.1002/ente.201300072.

(23) Imahara, H.; Minami, E.; Saka, S. Thermodynamic Study on Cloud Point of Biodiesel with Its Fatty Acid Composition. Fuel 2006, 85 (12-13), 1666-1670. https://doi.org/10.1016/j.fuel.2006.03.003.

(24) Acharya, N.; Nanda, P.; Panda, S.; Acharya, S. Analysis of Properties and Estimation of Optimum Blending Ratio of Blended Mahua Biodiesel. Eng. Sci. Technol. an Int. J. 2017, 20 (2), 511-517. https://doi.org/10.1016/j.jestch.2016.12.005. 
(25) Salaheldeen, M.; Aroua, M. K.; Mariod, A. A.; Foon, S.; Abdelrahman, M. A.; Atabani, A. E. Physicochemical Characterization and Thermal Behavior of Biodiesel and Biodiesel Diesel Blends Derived from Crude Moringa Peregrina Seed Oil. Energy Convers. Manag. 2015, 92, 535-542. https://doi.org/10.1016/j.enconman.2014.12.087.

(26) Pérez, Á.; Casas, A.; Fernández, C. M.; Ramos, M. J.; Rodríguez, L. Winterization of Peanut Biodiesel to Improve the Cold Flow Properties. Bioresour. Technol. 2010, 101 (19), 7375-7381. https://doi.org/10.1016/j.biortech.2010.04.063.

(27) Moser, B. R.; Vaughn, S. F. Evaluation of Alkyl Esters from Camelina Sativa Oil as Biodiesel and as Blend Components in Ultra Low-Sulfur Diesel Fuel. Bioresour. Technol. 2010, 101 (2), 646-653. https://doi.org/10.1016/j.biortech.2009.08.054.

(28) Rashid, U.; Ibrahim, M.; Nehdi, I. A.; Al-Resayes, S. I.; Ullah, S.; Mehmood, M. A.; Shahzadi, S. Synthesis and Characterization of Poppy Seed Oil Methyl Esters. Chinese J. Chem. Eng. 2016, 24 (8), 1087-1096. https://doi.org/10.1016/j.cjche.2016.01.007.

(29) Bhuiya, M. M. K.; Rasul, M. G.; Khan, M. M. K.; Ashwath, N. Biodiesel Production and Characterisation of Poppy (Papaver Somniferum L.) Seed Oil Methyl Ester as a Source of 2nd Generation Biodiesel Feedstock. Ind. Crops Prod. 2020, 152 (September 2019), 112493. https://doi.org/10.1016/j.indcrop.2020.112493.

(30) Sharafutdinov, I.; Stratiev, D.; Shishkova, I.; Dinkov, R.; Batchvarov, A.; Petkov, P.; Rudnev, N. Cold Flow Properties and Oxidation Stability of Blends of near Zero Sulfur Diesel from Ural Crude Oil and FAME from Different Origin. Fuel 2012, 96, 556-567. https://doi.org/10.1016/j.fuel.2011.12.062. 
(31) Ramadhas, A. S.; Jayaraj, S.; Muraleedharan, C. Biodiesel Production from High FFA Rubber Seed Oil. Fuel 2005, 84 (4), 335-340. https://doi.org/10.1016/j.fuel.2004.09.016.

(32) Mihaela, P.; Josef, R.; Monica, N.; Rudolf, Z. Perspectives of Safflower Oil as Biodiesel Source for South Eastern Europe (Comparative Study: Safflower, Soybean and Rapeseed). Fuel 2013, 111 (2013), 114-119. https://doi.org/10.1016/j.fuel.2013.04.012.

(33) Wakil, M. A.; Kalam, M. A.; Masjuki, H. H.; Atabani, A. E.; Rizwanul Fattah, I. M. Influence of Biodiesel Blending on Physicochemical Properties and Importance of Mathematical Model for Predicting the Properties of Biodiesel Blend. Energy Convers. Manag. 2015, 94, 51-67. https://doi.org/10.1016/j.enconman.2015.01.043.

(34) Cao, L.; Wang, J.; Liu, K.; Han, S. Ethyl Acetoacetate: A Potential Bio-Based Diluent for Improving the Cold Flow Properties of Biodiesel from Waste Cooking Oil. Appl. Energy 2014, 114, 18-21. https://doi.org/10.1016/j.apenergy.2013.09.050.

(35) Ranjan, A.; Dawn, S. S.; Jayaprabakar, J.; Nirmala, N.; Saikiran, K.; Sai Sriram, S. Experimental Investigation on Effect of MgO Nanoparticles on Cold Flow Properties, Performance, Emission and Combustion Characteristics of Waste Cooking Oil Biodiesel. Fuel 2018, 220 (August 2017), 780-791. https://doi.org/10.1016/j.fuel.2018.02.057.

(36) Bencheikh, K.; Atabani, A. E.; Shobana, S.; Mohammed, M. N.; Uğuz, G.; Arpa, O.; Kumar, G.; Ayanoğlu, A.; Bokhari, A. Fuels Properties, Characterizations and Engine and Emission Performance Analyses of Ternary Waste Cooking Oil Biodiesel-Diesel-Propanol Blends. Sustain. Energy Technol. Assessments 2019, 35 (September 2018), 321-334. https://doi.org/10.1016/j.seta.2019.08.007. 
(37) Evcil, A.; Al-Shanableh, F.; Savas, M. A. Variation of Solid Fraction with Cold Flow Properties of Biodiesel Produced from Waste Frying Oil. Fuel 2018, 215 (September 2017), 522-527. https://doi.org/10.1016/j.fuel.2017.11.055.

(38) Folayan, A. J.; Anawe, P. A. L.; Aladejare, A. E.; Ayeni, A. O. Experimental Investigation of the Effect of Fatty Acids Configuration, Chain Length, Branching and Degree of Unsaturation on Biodiesel Fuel Properties Obtained from Lauric Oils, High-Oleic and HighLinoleic Vegetable Oil Biomass. Energy Reports 2019, 5, 793-806. https://doi.org/10.1016/j.egyr.2019.06.013.

(39) Vijayan, S. K.; Naveena Victor, M.; Sudharsanam, A.; Chinnaraj, V. K.; Nagarajan, V. Winterization Studies of Different Vegetable Oil Biodiesel. Bioresour. Technol. Reports 2018, 1, 50-55. https://doi.org/10.1016/j.biteb.2018.02.005.

(40) Alptekin, E.; Canakci, M.; Sanli, H. Biodiesel Production from Vegetable Oil and Waste Animal Fats in a Pilot Plant. Waste Manag. 2014, 34 (11), 2146-2154. https://doi.org/10.1016/j.wasman.2014.07.019.

(41) Mofijur, M.; Masjuki, H. H.; Kalam, M. A.; Atabani, A. E. Evaluation of Biodiesel Blending, Engine Performance and Emissions Characteristics of Jatropha Curcas Methyl Ester: Malaysian Perspective. Energy 2013, 55, 879-887. https://doi.org/10.1016/j.energy.2013.02.059.

(42) Lapuerta, M.; Rodríguez-Fernández, J.; Fernández-Rodríguez, D.; Patiño-Camino, R. Cold Flow and Filterability Properties of N-Butanol and Ethanol Blends with Diesel and Biodiesel Fuels. Fuel 2018, $224 \quad$ (January), 552-559. 
https://doi.org/10.1016/j.fuel.2018.03.083.

(43) Lv, P.; Cheng, Y.; Yang, L.; Yuan, Z.; Li, H.; Luo, W. Improving the Flow Properties of Palm Oil Biodiesel: Addition of Cold Flow Improver. Fuel Process. Technol. 2013, 110, 61-64. https://doi.org/10.1016/j.fuproc.2012.12.014.

(44) Soriano, N. U.; Migo, V. P.; Matsumura, M. Ozonized Vegetable Oil as Pour Point Depressant for Neat Biodiesel. Fuel 2006, 85 (1), 25-31. https://doi.org/10.1016/j.fuel.2005.06.006.

(45) Santos, E. M.; Piovesan, N. D.; De Barros, E. G.; Moreira, M. A. Low Linolenic Soybeans for Biodiesel: Characteristics, Performance and Advantages. Fuel 2013, 104, 861-864. https://doi.org/10.1016/j.fuel.2012.06.014.

(46) Anwar, M.; Rasul, M.; Ashwath, N. Optimization of Biodiesel Production from Stone Fruit Kemel Oil. Energy Procedia 2019, 160 (2018), 268-276. https://doi.org/10.1016/j.egypro.2019.02.146. 